\title{
Efficacy of biological disease-modifying antirheumatic drugs: a systematic literature review informing the 2016 update of the EULAR recommendations for the management of rheumatoid arthritis
}

\author{
Jackie L Nam, ${ }^{1,2}$ Kaoru Takase-Minegishi, ${ }^{3}$ Sofia Ramiro, ${ }^{4}$ Katerina Chatzidionysiou, ${ }^{5}$ \\ Josef S Smolen, ${ }^{6,7}$ Désirée van der Heijde, ${ }^{4}$ Johannes W Bijlsma, ${ }^{8}$ Gerd R Burmester, ${ }^{9}$ \\ Maxime Dougados, ${ }^{10}$ Marieke Scholte-Voshaar, ${ }^{11,12}$ Ronald van Vollenhoven, ${ }^{13,14}$ \\ Robert Landewé ${ }^{13,15}$
}

- Additional material is published online only. To view please visit the journal online (http://dx.doi.org/10.1136/ annrheumdis-2016-210713).

For numbered affiliations see end of article.

Correspondence to Dr Jackie L Nam, Department of Rheumatology, Second Floor, Chapel Allerton Hospital, Chapeltown Road, Leeds LS7 4SA, UK; J.Nam@leeds.ac.uk

Received 22 October 2016 Revised 12 January 2017 Accepted 19 February 2017 Published Online First 3 May 2017

\section{Linked}

http://dx.doi.org/10.1136/ annrheumdis-2016-210715

- http://dx.doi.org/10.1136/ annrheumdis-2016-211005

CrossMark

To cite: Nam JL, TakaseMinegishi K, Ramiro S, et al. Ann Rheum Dis 2017:76:1108-1113.

\section{ABSTRACT}

Objectives To update the evidence for the efficacy of biological disease-modifying antirheumatic drugs (bDMARDs) in patients with rheumatoid arthritis (RA) to inform European League Against Rheumatism (EULAR) Task Force treatment recommendations.

Methods MEDLINE, EMBASE and Cochrane databases were searched for phase III or IV (or phase II, if these studies were lacking) randomised controlled trials (RCTs) published between January 2013 and February 2016. Abstracts from the American College of Rheumatology and EULAR conferences were obtained.

Results The RCTs confirmed greater efficacy with a bDMARD+conventional synthetic DMARD (csDMARD) versus a csDMARDs alone (level 1A evidence). Using a treat-to-target strategy approach, commencing and escalating csDMARD therapy and adding a bDMARD in cases of non-response, is an effective approach (1B). If a bDMARD had failed, improvements in clinical response were seen on switching to another bDMARD (1A), but no clear advantage was seen for switching to an agent with another mode of action. Maintenance of clinical response in patients in remission or low disease activity was best when continuing rather than stopping a bDMARD, but bDMARD dose reduction or 'spacing' was possible, with a substantial proportion of patients achieving bDMARD-free remission (2B). RCTs have also demonstrated efficacy of several new bDMARDs and biosimilar DMARDs (1B).

Conclusions This systematic literature review consistently confirmed the previously reported efficacy of bDMARDs in RA and provided additional information on bDMARD switching and dose reduction.

\section{INTRODUCTION}

Since the 2013 systematic literature review (SLR) on biological disease-modifying antirheumatic drugs (bDMARDs) in rheumatoid arthritis (RA), there have been several trials addressing efficacy and safety of various established bDMARDs, looking at different aspects of therapy including induction, switching, tapering and stopping of bDMARDs. There have also been publications on new bDMARDs, including some with new modes of action, as well as on a number of biosimilar DMARDs (bsDMARDs).

Many clinical trials provide direct comparisons between a bDMARD and a conventional synthetic DMARD (csDMARD). The use of treat-to-target strategies, ${ }^{2}$ however, better reflects real-life treatment approaches and therefore provides additional evidence for the use of these therapies in clinical practice. This SLR therefore also sought to provide an update on bDMARD strategy studies, previously defined as 'clinical trial(s) of any treatment of RA in which at least one arm consists of medication adjustment according to protocol, based on clinical outcomes aiming at a specific target'. ${ }^{3}$

This SLR aimed to update the body of evidence with information that has emerged since 2013 regarding the use of bDMARDs in RA. The results of this SLR and two others ${ }^{4} 5$ provided the task force with the current state of evidence.

\section{METHODS}

The updated standard operating procedures by European League Against Rheumatism (EULAR) were followed. ${ }^{6}$ As before, ${ }^{17}$ studies on the following nine bDMARDs were included: adalimumab (ADA), certolizumab-pegol (CZP), etanercept (ETN), golimumab (GLM), infliximab (IFX), anakinra (ANA), abatacept (ABT), rituximab (RTX) and tocilizumab (TCZ). ${ }^{1}{ }^{7}$ Information was also sought on new bDMARDs, including bsDMARDs. The search was performed using MEDLINE, EMBASE and Cochrane CENTRAL databases between January 2013 and February 2016. Relevant abstracts were sought from the 2013-2015 American College of Rheumatology (ACR) and 2014-2016 EULAR conferences.

The study selection criteria were the same as those in previous EULAR bDMARD SLRs. ${ }^{17}$ The Cochrane risk of bias assessment tool for RevMan $5.1^{8}$ was used to assess the quality of published studies and the Oxford Centre for Evidence-based Medicine levels of evidence ${ }^{9}$ was used to assign 
levels of evidence. Details on the search strategy can be found in the online supplementary material.

\section{RESULTS}

Of 10187 articles from the database search, together with additional ACR and EULAR conference abstracts and articles found after the database search, 51 published papers and 35 abstracts met the inclusion criteria. Risk of bias was considered 'low' for most but not all studies. Open-label trials were assigned 'high risk of bias' for the category 'blinding of participants and personnel' (see online supplementary material).

Efficacy data are presented in five sections: (1) bDMARD efficacy trials (in combination with a csDMARD or as monotherapy); (2) bDMARD strategy trials; (3) bDMARD switching trials; (4) bDMARD stopping or dose reduction trials and (4) trials with new therapies (new bDMARDs and bsDMARDs, and bDMARDs versus a targeted synthetic DMARD (tsDMARD)).

Patients with RA were grouped as follows: (1) DMARD-naive, (2) methotrexate (MTX)-naive, (3) MTX-inadequate response (IR), (4) csDMARD-IR (mixed DMARD-IR) and (5) tumor necrosis factor inhibitor (TNFi) TNFi-IR. This is highlighted for each study and studies are divided accordingly in the online supplementary section.

\section{Biological DMARD efficacy}

The focus of the results was on the primary outcomes. Other efficacy outcomes are presented in the online supplementary section.

\section{Existing biological DMARD+CsDMARD combination versus CSDMARD}

Nine new studies have been published after 2013 confirming evidence for the efficacy of a bDMARD + csDMARD versus a csDMARD. ${ }^{10-18}$ In DMARD-naive RA (2010 ACR/EULAR ${ }^{19}$ ), the C-EARLY ${ }^{10}$ study met its primary endpoint of sustained Disease Activity Score using a 28 joint count (DAS28) $<2.6$ between weeks 40 and 52 (CZP+MTX vs placebo+MTX: 29\% vs $15 \%$ ). In MTX-naive RA, C-OPERA confirmed better efficacy of CZP+MTX compared with MTX alone. ${ }^{14}$ CARDERA-2 failed to demonstrate radiological superiority of ANA+MTX versus MTX monotherapy. ${ }^{13}$ In mixed DMARD-IR patients, subcutaneous TCZ+MTX was superior to background MTX in the BREVACTA study (ACR 20 at week 24: 61\% vs 32\% at week 24), ${ }^{17}$ and RTX+background leflunomide to leflunomide in the AMARA study. ${ }^{18}$

The results of these new randomised controlled trials (RCTs) are in accordance with the previously formulated standpoint that a combination of a bDMARD and a csDMARD is more effective than a csDMARD alone. Level of evidence (LOE) as in the previous SLRs: ${ }^{1}$ 1A.

\section{Biological DMARD+MTX combination versus biological DMARD monotherapy}

In the MTX-naive RA AVERT study, a status of DAS2 $8<2.6$ was more often achieved with ABT+MTX than with MTX monotherapy or ABT monotherapy at 12 months (60.9\% vs $45.2 \%$ vs $42.5 \%)$. The FUNCTION study also showed higher proportions of patients with DAS remission and ACR responses-and less radiographic progression-with TCZ $8 \mathrm{mg} / \mathrm{kg}+$ MTX compared with TCZ monotherapy. ${ }^{20} 21$ TCZ monotherapy had more DAS28<2.6 and less radiographic progression than MTX monotherapy, but most other secondary endpoints, including physical function, were not different.
In MTX- IR RA patients, the SURPRISE study showed at week 24 , the time of the primary endpoint, that a status of DAS2 $8<2.6$ was more often achieved when adding TCZ to MTX versus switching from MTX to TCZ (70\% vs 55\%). ${ }^{22}$ This modest benefit had disappeared at week 52 (72\% vs $70 \%)$. Clinically relevant radiographic progression was lower with TCZ+MTX combination therapy than with TCZ monotherapy (van der Heijde-Sharp score $\geq 3$ : $7 \%$ vs $15 \%$ ).

The results of the newer RCTs are in accordance with the previously formulated standpoint that a combination of any bDMARD and a csDMARD is more effective than bDMARD monotherapy (LOE as in the previous SLRs: ${ }^{1} 1 \mathrm{~B}$ ).

\section{Biological strategy-type studies}

In the U-Act-Early RCT, MTX-naive patients were randomised to TCZ+MTX, TCZ monotherapy or MTX monotherapy using a treat-to-target approach. ${ }^{23}$ The primary analysis (number of people achieving sustained DAS $28<2.6$ by the originally assigned treatment) was higher in the TCZ+MTX or TCZ monotherapy groups than the MTX monotherapy group (86\% vs $84 \%$ vs $44 \%$ ). In the clinically more relevant second analysis, and co-primary endpoint, which addresses the entire study period, the initial differences between the groups were no longer seen with the addition of TCZ in the MTX monotherapy group following a treat-to-target approach $(86 \%$ vs $88 \%$ vs $77 \%)$.

In TACIT, a non-inferiority RCT in MTX-IR RA who had failed MTX and another csDMARD, ${ }^{24}$ patients were randomised to either a strategy of TNFi-start, followed by a switch to a second bDMARD in case of no response, or to a strategy of combination csDMARD therapy, followed by the start of a bDMARD in case of non-response. The change in Health Assessment Questionnaire (HAQ) score after 12 months (primary endpoint) was not inferior for the strategy starting with combination csDMARD versus the strategy starting with TNFi $(-0.45$ vs -0.3$)$. While earlier clinical responses were seen in the TNFi strategy, a status of DAS2 $<2.6$ at 12 months was met by slightly more patients in the TNF start group than in the csDMARD group ( $44 \%$ vs $35 \%$ ). Of note this was an open-label study with a (too) large non-inferiority margin that importantly limits its interpretability. Thus, the study had a high risk of bias. Adverse events were more frequently found in the csDMARD combination group.

Ten-year data from all four arms of the BeSt trial suggested that a high proportion of patients (53\%) maintain long-term remission, either on drugs or drug free, and had very limited 10-year radiographic progression, confirming the effectiveness of early DMARD treatment together with a treat-to-target approach. $^{25}$

The results of the newer RCTs are therefore in accordance with the previously formulated standpoint that strategies aiming at benchmarking disease activity and intensifying treatment when clinical remission or low disease activity is not yet reached may lead to favourable outcomes (LOE: 1B).

\section{Switching between bDMARDs in TNFi-IR RA}

Previous meta-analyses of RCTs had already demonstrated efficacy of all bDMARD classes in patients failing a TNFi (TNFi-IR) (LOE: 1A). ${ }^{7}$ To date, new bDMARD switching trials of this type could not be found.

Patients from the DREAM cohort, who had failed a first TNFi and had DAS28 $\geq 3.2$, were randomised to receive ABT or RTX, or a second TNFi in a trial with a non-inferiority design. The mean (SD) 12-month DAS28 were 3.8 (1.2) versus 3.4 (1.2) versus $3.5(1.5)$ in the ABT, RTX and TNFi groups, 
respectively. ${ }^{27}$ In the ROC trial, patients who failed their first TNFi were randomised to either a second TNFi or to another mode of action bDMARD (ABT, RTX or TCZ). ${ }^{28}$ At week 48, EULAR good response was $60 \%$ with a non-TNFi bDMARD versus $43.2 \%$ for a second TNFi.

The results of the newer RCTs are in accordance with the previously formulated standpoint that patients who have failed their first TNFi may expect benefit from a second TNFi or from a non-TNFi biological (LOE: 1A). There is insufficient evidence to prioritise either strategy.

\section{Biological DMARD stopping or dose reduction Biological DMARD stopping}

In patients with MTX-naive RA, the AVERT trial ${ }^{11}$ showed that patients with DAS2 $8<3.2$ on ABT +MTX, ABT or MTX maintained their drug-free status (DAS28 $<2.6$, both at 12 and 18 months) in only $14.8 \%$ after stopping ABT +MTX, $12.4 \%$ after stopping $\mathrm{ABT}$ and $7.8 \%$ after stopping MTX.

In patients with MTX-IR RA, in the ENCOURAGE study, patients with DAS2 $8<2.6$ on ETN+MTX at 6 and 12 months were randomly assigned to strategies stopping or continuing their treatment. There were higher proportions of patients with DAS28 $<2.6$ when continuing medication $(88 \%)$ versus withdrawing ETN and continuing MTX (54\%). ${ }^{29}$

In patients with MTX-IR RA that had participated in the ACT-RAY study, a follow-up study showed that in those with sustained DAS28 $<2.6$ and discontinued TCZ only $38.4 \%$ of the TCZ+MTX group and $35.1 \%$ of the TCZ monotherapy group maintained that state for an average of 3 months. ${ }^{30}$ The majority of those who lost response (84\%) responded well to TCZ reintroduction, but $16 \%$ did not.

The results of the newer RCTs are in accordance with the previously formulated standpoint that a variable but relatively low proportion of patients who have sustained low disease activity or remission on a strategy with a bDMARD can stop that bDMARD (and continue MTX) without losing their status of low disease activity/remission (LOE: 2B).

\section{Biological DMARD dose reduction}

In MTX-naive RA patients, in a substudy of AGREE, patients with a DAS28 erythrocyte sedimentation rate (DAS28-ESR) $<2.6$ at year 2 on ABT $10 \mathrm{mg} / \mathrm{kg}+$ MTX were randomised to ABT $10 \mathrm{mg} / \mathrm{kg}$ (full dose)+MTX versus ABT $5 \mathrm{mg} / \mathrm{kg}$ (half dose)+MTX. ${ }^{31}$ Similar relapse rates were seen in both groups (31\% in the ABT $10 \mathrm{mg} / \mathrm{kg}$ and $34 \%$ in the $5 \mathrm{mg} / \mathrm{kg}$ groups).

The open-label non-inferiority DRESS RCT, in which patients in stable low disease activity on ADA or ETN were randomised to usual care or a dose reduction strategy (stepwise increase in injection intervals), showed that continuation versus dose reduction led to similar rates of 'major flare' $(10 \%$ vs $12 \%) .^{32}$

In the OPTIRRA RCT, patients in stable (3 months) low disease activity (DAS28<3.2) on ADA or ETN were randomised to continue ADA or ETN, taper ADA or ETN by 33\% or taper ADA or ETN by $66 \% .{ }^{33}$ Similar flare rates were seen in the continuation and ADA or ETN 33\% tapering group (14\% vs 13\%), but a higher rate in the ADA or ETN 66\% tapering group (37\%).

The SMART ${ }^{34}$ study, in which TNFi-IR RA patients who achieved a EULAR (moderate or good) response on standard dose RTX were randomised to receive RTX $1000 \mathrm{mg}$ once or RTX $1000 \mathrm{mg}$ twice, suggested non-inferiority of both strategies (adjusted mean difference in DAS28-C reactive protein (DAS28CRP) area under the curve 51.4 (95\% CI -13.2 to 234)).

The results of the newer RCTs are in accordance with the previously formulated standpoint that a significant proportion of patients who have sustained low disease activity on a strategy with a bDMARD can taper that bDMARD (and continue MTX) without losing their status of low disease activity and that reducing the dose of the bDMARD by up to $50 \%$ or increasing the interval between doses accordingly conveys similar results as continuing full dose (LOE: 2B).

\section{bDMARDs in comparison to new therapies} Existing bDMARDs versus new targeted synthetic DMARDs

In the MTX-IR RA-BEAM study, comparing ADA+MTX versus the tsDMARD baricitinib+MTX versus placebo+MTX, showed small but significantly lower responses for ADA+MTX versus baricitinib+MTX, but both were higher than placebo+MTX (DAS28-CRP $<2.619 \%$ vs $24 \%$ vs $4 \%$ ) at week $12 .{ }^{35}$

\section{New biological DMARDs}

Several new bDMARDs targeting well-known targets have undergone phase II or III clinical trials in MTX-IR or mixed-DMARD-IR RA patients and have consistently shown superiority in clinical responses versus placebo. These include the human interleukin (IL)-6-receptor-inhibitor sarilumab, ${ }^{36}$ the humanised anti-IL6 clazakizumab, ${ }^{37}$ the human anti-IL6 siruku$\mathrm{mab}^{38}$ and also the granulocyte-monocyte colony stimulating factor receptor alpha inhibitor (GMCSFro-i) mavrilimumab. ${ }^{39}$ On the other hand, bDMARDs targeting the IL12/ 23 p40-pathway (ustekinumab), ${ }^{40}$ the IL23p19-pathway (guselkumab) ${ }^{40}$ and the B-cell-activating factor (tabalumab) ${ }^{41-43}$ have not demonstrated clinical efficacy over placebo in RA.

Studies have also formally demonstrated efficacy for siruku$\mathrm{mab}^{38}$ and sarilumab ${ }^{44}$ in patients previously exposed to other bDMARDs.

\section{Biosimilar DMARDs}

The long-term observational study of the PLANETRA trial has suggested sustained efficacy of those treated with the bsDMARD IFX CT-P13. ${ }^{45}$ IFX CT-P13 also demonstrated clinical efficacy in another RCT of MTX-IR RA. ${ }^{46}$ Efficacy was also formally proven in placebo-controlled RCTs with the ADA bsDMARDs ABP50 $1^{47}$ and SB5, ${ }^{48}$ with the ETN bsDMARDs $\mathrm{HD} 203^{49}$ and SB4, ${ }^{50}$ with the IFX bsDMARD SB2 ${ }^{51}$ and with the RTX bsDMARD BCD-020. ${ }^{52}$

The results of the newer RCTs are in accordance with the previously formulated standpoint that targeting the IL6-pathway, now including also the IL-6 ligand, may provide benefits to patients, that targeting the cytokine GMCSF is potentially beneficial to patients and that bsDMARDs are as effective biologicals as the originator bDMARDs in the treatment of patients with RA.

\section{DISCUSSION}

This review on bDMARDs in RA aimed to provide a systematic update of the body of evidence available for the treatment of patients with RA with bDMARDs. It only includes new data from 2013 onwards. These data were presented to the expert committee that convened to discuss the 2016 update of the EULAR recommendations on the (drug) management of patients with RA. ${ }^{53}$

The results of this SLR confirmed the efficacy of bDMARDs in combination with a csDMARD (ADA, CZP, ETN, GLM, IFX, ABT, RTX and TCZ but not ANA). ${ }^{13}$ Combination therapy (bDMARD+csDMARD) was in general again found to be superior to bDMARD monotherapy.

Remarkably, we did not find any new 'head-to-head' trial with bDMARDs published after 2013 in this highly competitive field of high-cost drug treatment in RA. Investigators of 
sponsored trials usually sought (reconfirmation of) superiority over placebo or engaged in low-commercial-risk strategy trials that reconfirmed the already inarguable efficacy of their bDMARD over placebo.

What is needed in the field of RA, known for its high number of very effective but costly treatments, is a proper evidence-based prioritisation of the drugs we have available. Guideline committees such as ours have to base their consensus on solid data stemming from direct comparisons of treatments. In the absence of high-quality direct comparisons, methodologists and (company) statisticians find escape routes in indirect comparisons and network meta-analyses. We will not dispute the modest merits of network meta-analyses, but warn against the careless interpretation of their results, since no (network) meta-analysis is methodologically better than the weakest trial contributing to it.

The most important findings in this update SLR were as follows: patients on MTX monotherapy achieved sustained remission when following a treat-to-target strategy. ${ }^{23}$ Results from new strategy studies ${ }^{23} 24$ in this regard support those from previous $\mathrm{RCTs}^{13}$ and allow a firm conclusion: a treat-to-target approach, escalating csDMARD therapy and adding a bDMARD in cases of non-response, is an effective approach.

New trials in patients who have failed their first TNFi show that switching to a second bDMARD 'makes sense'. However, the current RCTs do not help us in deciding if this second bDMARD should be a TNFi DMARD or a non-TNFi DMARD. Sparse data that are currently available are not convincing. It may, for instance, be relevant that a patient has not had any response to bDMARD from its initiation (primary nonresponse) or that an initial response was lost over time (secondary non-response). Evidence from RCTs that may help answering such questions is still lacking.

Recently we have faced the advent of several bsDMARDs. Many of these have passed the hurdle of regulatory 'biosimilarity' and have entered the market or will do this soon. To date, there is no scientific indication that these bsDMARDs, which are already less expensive in some countries than their originator counterparts, are inferior to their 'parents' in efficacy or safety. In the absence of tangible distinctions between originator bDMARDs and their bsDMARDs, future guideline committees will likely base their priority on non-scientific arguments such as drug costs.

In general, patients with RA that have achieved low disease activity or remission are better off with continuation of their treatment than with stopping, but many of the patients can successfully apply bDMARD dose reduction or interval increase, and if a flare occurs most of them will regain disease control upon restarting their bDMARD. Prognostic factors that may help determining which patient subgroups are able to de-escalate therapy and achieve drug-free remission are needed. ${ }^{54}$ Several studies have addressed these ${ }^{55-58}$ but we could not find RCTs in which patients had been stratified according to prognostic factors for tapering.

Comparative data with the tsDMARD baricitinib suggested superior efficacy of baricitinib over ADA, but it remains to be seen if this short-term gain remains over time. Obviously, longterm data on safety still have to be awaited before a proper valuation can take place. There were also several RCTs demonstrating efficacy of new mode-of-action bDMARDs and bsDMARDs in RA.

As before, the sole source of efficacy studies in this SLR was RCTs. While registry data may provide real-life efficacy data, these are prone to bias and have not been included in this SLR. Registry data, however, are crucial to evaluate long-term drug safety and have been used in the EULAR SLR addressing DMARD safety. ${ }^{4}$

In conclusion, this literature review consistently confirmed the efficacy of bDMARDs in RA. It provides some evidence for bDMARD stopping and dose reduction, addressed the important topic of bDMARD switching in TNFi-IR RA and highlighted the advent of some new biological therapies.

\section{Author affiliations}

${ }^{1}$ Leeds Institute of Rheumatic and Musculoskeletal Medicine, University of Leeds, Leeds, UK

${ }^{2}$ NIHR Leeds Musculoskeletal Biomedical Research Unit, Leeds Teaching Hospitals NHS Trust, Leeds, UK

${ }^{3}$ Center for Rheumatic Diseases, Yokohama City University Medical Center, Yokohama, Japan

${ }^{5}$ Unit for Clinical Therapy Research, Inflammatory Diseases (ClinTRID), The Karolinska Institute, Stokholm, Sweden

${ }^{6}$ Division of Rheumatology, Department of Medicine 3, Medical University of Vienna, Vienna, Austria

${ }^{7}$ Department of Medicine, Hietzing Hospital, Vienna, Austria

${ }^{8}$ Department of Rheumatology, University Medical Center Utrecht, Utrecht, The Netherlands

${ }^{9}$ Department of Rheumatology, Charité University Medicine Berlin, Berlin, Germany

${ }^{10}$ Department of Rheumatology, Paris Descartes University, Cochin Hospital,

Assistance Publique-Hôpitaux de Paris, INSERM (U1153): Clinical Epidemiology and

Biostatistics, Paris, France

${ }^{11}$ Department of Psychology, Health and Technology, University of Twente, Enschede, The Netherlands

${ }^{12}$ EULAR Standing Committee of People with Arthritis/Rheumatism in Europe

${ }^{13}$ Department of Clinical Immunology \& Rheumatology, Amsterdam Rheumatology

Center, Amsterdam, The Netherlands

${ }^{14}$ Department of Rheumatology, Amsterdam Rheumatology Center, Amsterdam,

The Netherlands

${ }^{15}$ Department of Rheumatology, Zuyderland Medical Center, Heerlen, The Netherlands

Correction notice This article has been corrected since it published Online First. Reference 8 has been updated.

\section{Acknowledgements}

The authors thank Liz Dalton, University of Leeds, for her expertise and help with the literature search and EULAR for funding this work.

Contributors JLN and KT extracted the data for the SLR. All coauthors contributed to the writing of the manuscript.

Competing interests JS: Amgen, Abbvie, Astra-Zeneca, Astro, BMS, Celgene, Glaxo, ILTOO, Janssen, Merck-Serono, MSD, Novartis-Sandoz, Pfizer, Roche-Chugai, Samsung, UCB. DvdH: AbbVie, Amgen, Astellas, AstraZeneca, BMS, Celgene, Daiichi, Eli-Lilly, Galapagos, Gilead, Merck, Novartis, Pfizer, Roche, Sanofi-Aventis, UCB, 5, Director of Imaging Rheumatology BV. JWB: Roche, AbbVie, Bristol-Myers Squibb, Merck Sharp \& Dohme, Pfizer, UCB. GB: UCB, AbbVie, BMS, Hexal, Janssen, Lilly, MSD, Medimmune, Novartis, Pfizer, Sanofi, Roche. MD, AbbVie, Pfizer, Novartis, MSD. RvV: AbbVie, Bristol-Myers Squibb, GlaxoSmithKline, Pfizer, Roche, UCB Pharma, Biotest, Janssen, Eli-Lilly, Merck, Vertex. RL: AbbVie, Amgen, Centocor, Novartis, Pfizer, Roche, Schering-Plough, UCB, Pfizer, Ablynx, Amgen, Astra-Zeneca, Bristol Myers Squibb, Celgene, Janssen (formerly Centocor), Galapagos, Glaxo-Smith-Kline, Novartis, Novo-Nordisk, Merck, TiGenix, Rheumatology Consultancy BV.

Provenance and peer review Not commissioned; externally peer reviewed.

\section{REFERENCES}

1 Nam JL, Ramiro S, Gaujoux-Viala C, et al. Efficacy of biological disease-modifying antirheumatic drugs: a systematic literature review informing the 2013 update of the EULAR recommendations for the management of rheumatoid arthritis. Ann Rheum Dis 2014;73:516-28.

2 Smolen JS, Breedveld FC, Burmester GR, et al. Treating rheumatoid arthritis to target: 2014 update of the recommendations of an international task force. Ann Rheum Dis 2016;75:3-15.

3 Knevel R, Schoels M, Huizinga TW, et al. Current evidence for a strategic approach to the management of rheumatoid arthritis with disease-modifying antirheumatic drugs: a systematic literature review informing the EULAR recommendations for the management of rheumatoid arthritis. Ann Rheum Dis 2010;69:987-94. 
4 Ramiro S, Sepriano A, Chatzidionysiou K, et al. Safety of synthetic and biological DMARDs - a systematic literature review informing the 2016 update of the EULAR recommendations for management of rheumatoid arthritis. Ann Rheum Dis 2017. doi: 10.1136/annrheumdis-2016-210708.

5 Chatzidionysiou K, Sharzad E, Nam JL, et al. Efficacy and Safety of Glucocorticoids, Conventional and Targeted Synthetic Disease-Modifying Antirheumatic Drugs: a Systematic Literature Review and Meta-analysis Informing the 2016 update of The EULAR Recommendations for the Management of Rheumatoid Arthritis. Ann Rheum Dis 2017. doi: 10.1136/annrheumdis-2016-210711.

6 Dougados M, Betteridge N, Burmester GR, et al. EULAR standardised operating procedures for the elaboration, evaluation, dissemination, and implementation of recommendations endorsed by the EULAR standing committees. Ann Rheum Dis 2004:63:1172-6.

7 Nam JL, Winthrop KL, van Vollenhoven RF, et al. Current evidence for the management of rheumatoid arthritis with biological disease-modifying antirheumatic drugs: a systematic literature review informing the EULAR recommendations for the management of RA. Ann Rheum Dis 2010;69:976-86.

8 Cochrane Handbook for Systematic Reviews of Interventions Version 5.1.0 March 2011 [cited 2016 September ]. http://handbook.cochrane.org/

9 Oxford Centre for Evidence-based Medicine_Levels of evidence. March 2009 (cited September 2016). http://www.cebm.net/index.aspx?0=1025

10 Emery P, Bingham CO, Burmester GR, et al. The first study of certolizumab pegol in combination with methotrexate in DMARD-naive early rheumatoid arthritis patients led to sustained clinical response and inhibition of radiographic progression at 52 weeks: The C-early randomized, double-blind, controlled phase 3 study. Ann Rheum Dis 2015;74(Suppl 2):712.

11 Emery P, Burmester GR, Bykerk VP, et al. Evaluating drug-free remission with abatacept in early rheumatoid arthritis: Results from the phase $3 \mathrm{~b}$, multicentre, randomised, active-controlled AVERT study of 24 months, with a 12-month, double-blind treatment period. Ann Rheum Dis 2015;74:19-26.

12 Takeuchi T, Yamanaka H, Ishiguro N, et al. Adalimumab, a human anti-TNF monoclonal antibody, outcome study for the prevention of joint damage in Japanese patients with early rheumatoid arthritis: The HOPEFUL 1 study. Ann Rheum Dis 2014;73:536-43.

13 Scott IC, Ibrahim F, Simpson G, et al. A randomised trial evaluating anakinra in early active rheumatoid arthritis. Clin Exp Rheumatol 2016;34:88-93.

14 Atsumi T, Yamamoto K, Takeuchi T, et al. The first double-blind, randomised, parallel-group certolizumab pegol study in methotrexate-naive early rheumatoid arthritis patients with poor prognostic factors, C-OPERA, shows inhibition of radiographic progression. Ann Rheum Dis 2016;75:75-83.

$15 \mathrm{Kim} \mathrm{J}, \mathrm{Ryu} \mathrm{H}, \mathrm{Yoo} \mathrm{DH}$, et al. A clinical trial and extension study of infliximab in Korean patients with active rheumatoid arthritis despite methotrexate treatment. J Korean Med Sci 2013;28:1716-22.

16 Takeuchi T, Miyasaka N, Zang C, et al. A phase 3 randomized, double-blind, multicenter comparative study evaluating the effect of etanercept versus methotrexate on radiographic outcomes, disease activity, and safety in Japanese subjects with active rheumatoid arthritis. Modern Rheumatol 2013;23:623-33.

17 Kivitz $A$, Olech $E$, Borofsky $M$, et al. Subcutaneous tocilizumab versus placebo in combination with disease-modifying antirheumatic drugs in patients with rheumatoid arthritis. Arthritis Care Res 2014;66:1653-61.

18 Behrens F, Rossmanith T, Köhm M, et al. Rituximab in combination with leflunomide: results from a multicenter randomized placebo controlled investigator initiated clinical trial in active rheumatoid arthritis (AMARA-STUDY). Ann Rheum Dis 2016;75(Suppl 2):502.

19 Aletaha D, Neogi T, Silman AJ, et al. 2010 rheumatoid arthritis classification criteria: an American College of Rheumatology/European League Against Rheumatism collaborative initiative. Ann Rheum Dis 2010;69:1580-8.

20 Burmester GR, Rigby WF, van Vollenhoven RF, et al. Tocilizumab in early progressive rheumatoid arthritis: FUNCTION, a randomised controlled trial. Ann Rheum Dis 2016;75:1081-91.

21 Burmester G, Rigby W, Van Vollenhoven RF, et al. Tocilizumab combination therapy or monotherapy or methotrexate monotherapy in methotrexate-naive patients with early rheumatoid arthritis: 2-year clinical and radiographic results from a randomized, placebo-controlled trial. Arthritis Rheumatol 2014;66(Suppl 10): S811-12.

22 Kaneko Y, Atsumi T, Tanaka Y, et al. Comparison of adding tocilizumab to methotrexate with switching to tocilizumab in patients with rheumatoid arthritis with inadequate response to methotrexate: 52 -week results from a prospective, randomised, controlled study (SURPRISE study). Ann Rheum Dis 2016;75: 1917-23.

23 Bijlsma JW, Welsing PM, Woodworth TG, et al. Early rheumatoid arthritis treated with tocilizumab, methotrexate, or their combination (U-Act-Early): a multicentre, randomised, double-blind, double-dummy, strategy trial. Lancet 2016;388:343-55.

24 Scott DL, Ibrahim F, Farewell V, et al. Tumour necrosis factor inhibitors versus combination intensive therapy with conventional disease modifying anti-rheumatic drugs in established rheumatoid arthritis: TACIT non-inferiority randomised controlled trial. BMJ (Online) 2015:350:h1046.
25 Markusse IM, Akdemir G, Dirven L, et al. Long-term outcomes of patients with recent-onset rheumatoid arthritis after 10 years of tight controlled treatment: a randomized trial. Ann Intern Med 2016;164:523-31.

26 Schoels M, Aletaha D, Smolen JS, et al. Comparative effectiveness and safety of biological treatment options after tumour necrosis factor $\alpha$ inhibitor failure in rheumatoid arthritis: systematic review and indirect pairwise meta-analysis. Ann Rheum Dis 2012;71:1303-8.

27 Manders SH, Kievit W, Adang E, et al. Cost-effectiveness of abatacept, rituximab, and TNFi treatment after previous failure with TNFi treatment in rheumatoid arthritis: a pragmatic multi-centre randomised trial. Arthritis Res Ther 2015;17:134

28 Gottenberg JE, Brocq O, Perdriger A, et al. In the multicenter randomized controlled rotation or change Trial, a non-TNF targeted therapy has a higher efficacy than a second anti-tnf at 3, 6 and 12 months. Arthritis Rheumatol 2015;67(Suppl 10):3725-7.

29 Yamanaka H, Nagaoka S, Lee SK, et al. Discontinuation of etanercept after achievement of sustained remission in patients with rheumatoid arthritis who initially had moderate disease activity-results from the ENCOURAGE study, a prospective, international, multicenter randomized study. Mod Rheumatol 2016:26:651-61

30 Huizinga TWJ, Conaghan PG, Martin-Mola E, et al. Clinical and radiographic outcomes at 2 years and the effect of tocilizumab discontinuation following sustained remission in the second and third year of the ACT-RAY study. Ann Rheum Dis 2015;74:35-43.

31 Westhovens $R$, Robles $M$, Ximenes AC, et al. Maintenance of remission following 2 years of standard treatment then dose reduction with abatacept in patients with early rheumatoid arthritis and poor prognosis. Ann Rheum Dis 2015;74:564-8.

32 van Herwaarden N, van der Maas A, Minten MJ, et al. Disease activity guided dose reduction and withdrawal of adalimumab or etanercept compared with usual care in rheumatoid arthritis: open label, randomised controlled, non-inferiority trial. BMJ 2015;350:h1389.

33 Galloway JB, Kingsley G, Ma M, et al. Optimising treatment with TNF inhibitors in rheumatoid arthritis with different dose tapering strategies: The OPTTIRA trial. Ann Rheum Dis 2015;74(Suppl 2):706.

34 Mariette X, Rouanet S, Sibilia J, et al. Evaluation of low-dose rituximab for the retreatment of patients with active rheumatoid arthritis: a non-inferiority randomised controlled trial. Ann Rheum Dis 2014;73:1508-14.

35 Taylor PC, Keystone EC, Van Der Heijde D, et al. Baricitinib versus placebo or adalimumab in patients with active rheumatoid arthritis (RA) and an inadequate response to background methotrexate therapy: results of a phase 3 study. Arthritis Rheumatol 2015;67(Suppl 10):3927-31.

36 Genovese MC, Fleischmann R, Kivitz AJ, et al. Sarilumab Plus Methotrexate in Patients With Active Rheumatoid Arthritis and Inadequate Response to Methotrexate: Results of a Phase III Study. Ann Rheum Dis 2015;67:1424-37.

37 Weinblatt ME, Mease $P$, Mysler E, et al. The efficacy and safety of subcutaneous clazakizumab in patients with moderate-to-severe rheumatoid arthritis and an inadequate response to methotrexate: results from a multinational, phase IIb, randomized, double-blind, placebo/active-controlled, dose-ranging study. Ann Rheum Dis 2015:67:2591-600.

38 Thorne C, Karpouzas G, Takeuchi T, et al. Response and radiographic progression in biologic-naïve and biologic-experienced patients with rheumatoid arthritis treated with sirukumab. Ann Rheum Dis 2016;75(Suppl 2):723.

39 Burmester G, McInnes IB, Kremer JM, et al. Efficacy and safety/tolerability of mavrilimumab, a human GM-CSFRA monoclonal antibody in patients with rheumatoid arthritis. Arthritis Rheumatol 2014;66(Suppl 10):S1231-2.

40 Smolen J, Agarwal SK, llivanova E, et al. A phase 2 study evaluating the efficacy and safety of subcutaneously administered ustekinumab and guselkumab in patients with active rheumatoid arthritis despite treatment with methotrexate [abstract]. Ann Rheum Dis 2015;74(Suppl 2):76-7.

41 Genovese MC, Silverman GJ, Emery $\mathrm{P}$, et al. Efficacy and safety of tabalumab, an anti-B-cell-activating factor monoclonal antibody, in a heterogeneous rheumatoid arthritis population: Results from a randomized, placebo-controlled, phase 3 trial (FLEX-O). J Clin Rheumatol 2015;21:231-8.

42 Schiff M, Combe B, Dörner T, et al. Efficacy and safety of tabalumab, an anti-BAFF monoclonal antibody, in patients with moderate-to-severe rheumatoid arthritis and inadequate response to TNF inhibitors: results of a randomised, double-blind, placebo-controlled, phase 3 study. RMD Open 2015;1:e000037.

43 Smolen JS, Weinblatt ME, Van Der Heijde D, et al. Efficacy and safety of tabalumab, an anti-B-cellactivating factor monoclonal antibody, in patients with rheumatoid arthritis who had an inadequate response to methotrexate therapy: Results from a phase III multicentre, randomised, double-blind study. Ann Rheum Dis 2015:74:1567-70.

44 Fleischmann R, Castelar-Pinheiro G, Brzezicki J, et al. Efficacy and safety of sarilumab in combination with csdmards in patients with active rheumatoid arthritis who were inadequate responders or intolerant of anti-TNF-+/- therapy: Results from a phase 3 study. Arthritis Rheumatol 2015;67(Suppl 10):1266-8.

45 Yoo DH, Prodanovic N, Jaworski J, et al. Efficacy and safety of CT-P13 (infliximab biosimilar) over two years in patients with rheumatoid arthritis: comparison between 
continued CT-P13 and switching from infliximab to CT-P13. Arthritis Rheum 2013;65:3319.

46 Takeuchi T, Yamanaka H, Tanaka Y, et al. Evaluation of the pharmacokinetic equivalence and 54-week efficacy and safety of CT-P13 and innovator infliximab in Japanese patients with rheumatoid arthritis. Modern Rheumatol 2015;25: $817-24$.

47 Matsumoto AK, Pavelka K, Rizzo W, et al. Secondary efficacy endpoints: Results from a phase 3 study comparing ABP 501 with adalimumab in subjects with moderate to severe rheumatoid arthritis [abstract]. Arthritis Rheumatol 2015;67 (Suppl 10):3331-2.

48 Weinblatt ME, Baranauskaite A, Niebrzydowski J, et al. A phase III, randomized, double-blind clinical study comparing SB5, an adalimumab biosimilar, with adalimumab reference product (humira) in patients with moderate to severe rheumatoid arthritis despite methotrexate therapy (24-week results). Arthritis Rheumatol 2015;67(Suppl 10):3946-8.

49 Bae SC, Kim J, Choe JY, et al. A phase III, multicentre, randomised, double-blind, active-controlled, parallel-group trial comparing safety and efficacy of HD203, with innovator etanercept, in combination with methotrexate, in patients with rheumatoid arthritis: the HERA study. Ann Rheum Dis 2017;76:65-71.

50 Emery P, Vencovsky J, Sylwestrzak A, et al. A phase III randomised, double-blind parallel-group study comparing SB4 with etanercept reference product in patients with active rheumatoid arthritis despite methotrexate therapy. Ann Rheum Dis 2017;76:51-7.

51 Choe JY, Prodanovic N, Niebrzydowski J, et al. A randomised, double-blind, phase III study comparing SB2, an infliximab biosimilar, to the infliximab reference product
Remicade in patients with moderate to severe rheumatoid arthritis despite methotrexate therapy. Ann Rheum Dis 2017;76:58-64.

52 Eremeeva A, Chernyaeva E, Ivanov $R$, et al. Comparison of efficacy and safety of rituximab biosimilar, bcd-020, and innovator rituximab in patients with active rheumatoid arthritis refractory to TNFA inhibitors. Ann Rheum Dis 2016;75(Suppl 2):513-14.

53 Smolen JS, Landewé R, Bijlsma JW, et al. EULAR recommendations for the management of rheumatoid arthritis with synthetic and biological disease-modifying antirheumatic drugs: 2016 update. Ann Rheum Dis 2017. doi: 10.1136/ annrheumdis-2016-210715. [Epub ahead of print 6 Mar 2017].

54 Schett G, Emery P, Tanaka Y, et al. Tapering biologic and conventional DMARD therapy in rheumatoid arthritis: current evidence and future directions. Ann Rheum Dis 2016:75:1428-37.

55 van der Woude D, Young A, Jayakumar K, et al. Prevalence of and predictive factors for sustained disease-modifying antirheumatic drug-free remission in rheumatoid arthritis: results from two large early arthritis cohorts. Arthritis Rheum 2009;60:2262-71.

56 Saleem B, Keen H, Goeb V, et al. Patients with RA in remission on TNF blockers: when and in whom can TNF blocker therapy be stopped? Ann Rheum Dis 2010;69:1636-42.

57 Tanaka Y, Hirata S, Kubo $S$, et al. Discontinuation of adalimumab after achieving remission in patients with established rheumatoid arthritis: 1-year outcome of the HONOR study. Ann Rheum Dis 2015;74:389-95.

58 Naredo E, Valor L, De la Torre I, et al. Predictive value of Doppler ultrasound-detected synovitis in relation to failed tapering of biologic therapy in patients with rheumatoid arthritis. Rheumatology (Oxford) 2015;54:1408-14. 